\title{
Nearby young stars ${ }^{\star}$
}

\author{
R. Wichmann ${ }^{1, \star \star, \star \star \star}$, J. H. M. M. Schmitt ${ }^{1}$, and S. Hubrig ${ }^{2}$ \\ 1 Hamburger Sternwarte, Gojenbergsweg 112, 21029 Hamburg, Germany \\ ${ }^{2}$ European Southern Observatory, Karl-Schwarzschild-Str. 2, 85748 Garching bei München, Germany
}

Received 4 October 2002 / Accepted 29 November 2002

\begin{abstract}
We present the results of an extensive all-sky survey of nearby stars of spectral type F8 or later in a systematic search of young (zero-age main sequence) objects. Our sample has been derived by cross-correlating the ROSAT All-Sky Survey and the TYCHO catalogue, yielding a total of 754 candidates distributed more or less randomly over the sky. Follow-up spectroscopy of these candidate objects has been performed on 748 of them. We have discovered a tight kinematic group of ten stars with extremely high lithium equivalent widths that are presumably younger than the Pleiades, but again distributed rather uniformly over the sky. Furthermore, about 43 per cent of our candidates have detectable levels of lithium, thus indicating that these are relatively young objects with ages not significantly above the Pleiades age.
\end{abstract}

Key words. surveys - Galaxy: solar neighbourhood - stars: kinematics - stars: late-type

\section{Introduction}

Traditionally, the study of zero-age main sequence (ZAMS) stars has been a study of open clusters and associations. However, there is some reason to expect ZAMS stars to also exist in the field, and in particular also in the solar neighbourhood. It is well known that tightly bound, long-lived open clusters can account for only a few per cent of the total galactic star formation rate (cf. Wielen 1971). The inescapable conclusion follows that either most clusters/associations disperse very quickly after star formation has started or that most stars are born in isolation.

Indeed a few very small associations are known, like the TW Hya association (Rucinski \& Krautter 1983; de la Reza et al. 1989; Gregorio-Hetem et al. 1992; Webb et al. 1999), the $\eta$ Chamaeleontis cluster (Mamajek et al. 1999), or the one in front of the translucent clouds MBM 7 and MBM 55

Send offprint requests to: $\mathrm{R}$. Wichmann, e-mail: rwichmann@hs. uni-hamburg.de

* Based on observations collected at the European Southern Observatory, Chile (ESO No. 62.I-0650, 66.D-0159(A), 67.D0236(A)).

$\star \star$ Visiting Astronomer, Kitt Peak National Observatory, National Optical Astronomy Observatory, which is operated by the Association of Universities for Research in Astronomy, Inc. (AURA) under cooperative agreement with the National Science Foundation.

$\star \star \star$ Visiting Astronomer, German-Spanish Astronomical Centre, Calar Alto, operated by the Max-Planck-Institute for Astronomy, Heidelberg, jointly with the Spanish National Commission for Astronomy.
(Hearty et al. 1999). We therefore prefer the first alternative, since there is little evidence for truly isolated star formation.

If then most star-forming associations dissociate rapidly, there must exist many rather young (i.e. ZAMS) stars in the field. Furthermore we have to keep in mind that the Sun and its neighbourhood is located within a huge, young star-forming complex known as the Gould Belt (GB; for a recent review of the GB see cf. Pöppel 1997). One therefore also expects that there are ZAMS field stars in the solar neighbourhood that have been formed in the GB over its lifetime and have dispersed into the field since then.

This latter view is supported by Guillout et al. (1998) who report the detection of an X-ray active, late-type stellar population associated with the GB, and apparently distributed in a "disrupted disk"-like structure. According to their model, young stars are not located exclusively at the outer edge of the GB, where most of its present-day star forming activity takes place, but their distribution extends into the inner parts of the GB.

This model is also supported by a study of lithium-rich stars detected towards the Lupus dark clouds (Wichmann et al. 1999). They were able to construct a distance distribution of these stars by combining $v \sin i$ data with photometrically determined rotational periods, and assuming a mean inclination angle of $\pi / 4$. This distance distribution (Fig. 8 in Wichmann et al. 1999) shows a peak at about the distance of the Lupus clouds, but also a marked tail extending to lower distances, i.e. inwards of the GB.

As a consequence one expects to find young stars also in the solar neighbourhood and the purpose of this paper is to report 


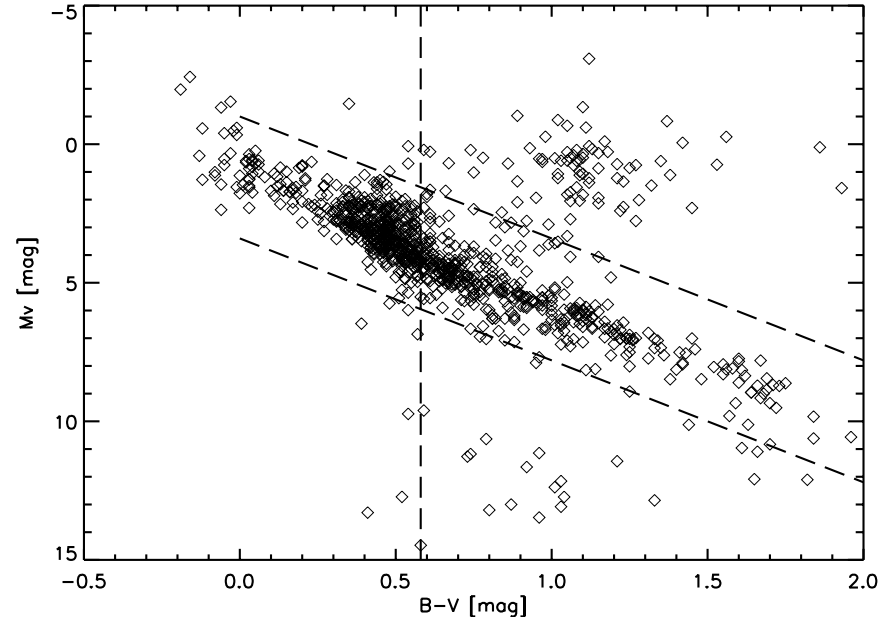

Fig. 1. H-R diagram showing the RASS-TYCHO sample and (dashed lines) our selection box.

on a systematic all-sky survey for young (ZAMS) stars in the solar vicinity, up to about $50 \mathrm{pc}$ distance.

\section{Candidate selection}

Since most young stars show strong X-ray emission, the candidate sample for this survey was selected from a crosscorrelation of the Rosat All-Sky Survey (RASS) with the TYCHO catalogue (ESA 1997a). We used the RassTych sample constructed by Guillout et al. (1999), and enhanced it ourselves by cross-correlating it with the HIPPARCOS catalogue (ESA 1997a). The resulting sample is similar to the RassHip sample shown in Fig. 5b of Guillout et al. (1999). From this sample, we selected stars with $(B-V)>0.54$ with (TYCHO) parallax errors better than $3.5 \sigma$. The cutoff at $(B-V)>0.54$ corresponds approximately to spectral type F8. We then proceeded by constructing an H-R diagram of the stars (Fig. 1), and discarded stars very far above the main sequence (giants) and very far below (erroneous parallaxes), resulting in a total sample size of 754 stars. Our candidate selection is visualized in Fig. 1, where the selection criteria and the resulting region in the $\mathrm{H}-\mathrm{R}$ diagram are outlined by dashed lines.

The restriction on spectral type was imposed mainly because there seems to be no reliable age indicator for earlier spectral types, as Li I is ionized away. The reason for the restriction on the parallax error was that we wanted to have some information on the location in the H-R diagram, and also on the $3 \mathrm{D}$ space velocity (which requires parallax information to convert proper motions into space velocities).

The distribution of Johnson $(B-V)_{J}$ colours in our sample is very similar to the one shown in Fig. 4 of Guillout et al. (1999). Because of the magnitude limit of the TYCHO catalogue, our sample is strongly biased towards G-type stars. This implies that in our survey we are likely to miss most of the young late-type stars in the field (assuming a standard IMF). In our sample, there are 473 stars earlier than $\mathrm{K} 0((B-V)<0.81)$, $257 \mathrm{~K}$-stars $(0.81 \leq(B-V)<1.4)$, and only 24 M-stars (in the range M0-M1.5).

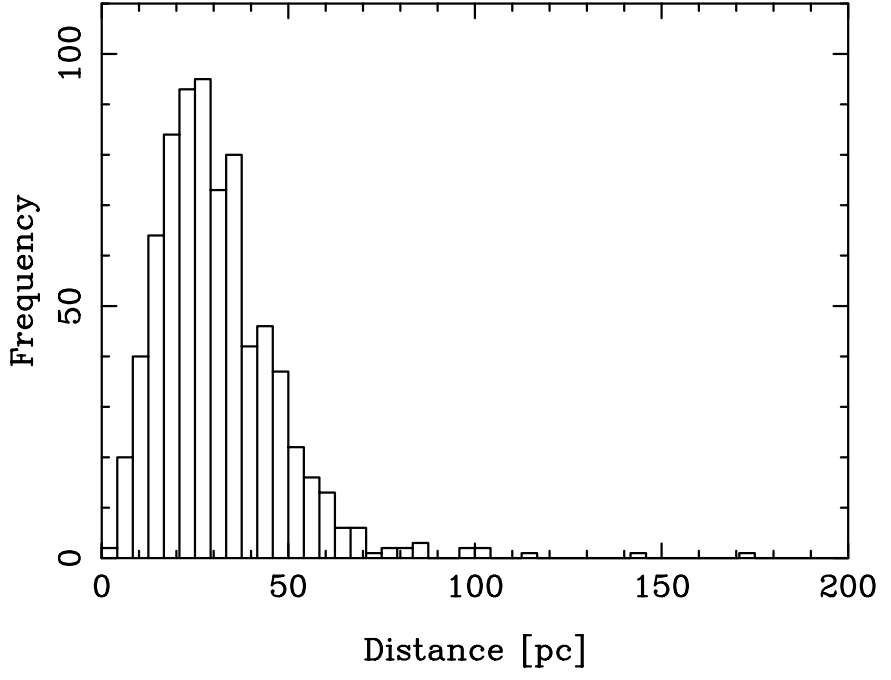

Fig. 2. Histogram showing the distribution of distances in our sample.

Likewise, the distribution of distances of our sample stars is shown in Fig. 2. If possible, parallaxes from HIPPARCOS (ESA 1997b) are used, otherwise we use TYCHO parallaxes (694 stars actually have HIPPARCOS parallaxes). The distance distribution (Fig. 2) has a peak at 20-30 pc, with a tail extending to about $200 \mathrm{pc}$. The few stars at very large distances are giants that were originally included in our sample as the result of incorrect TYCHO parallaxes, but Fig. 2 demonstrates clearly that the bulk of our sample stars is located closer than $50 \mathrm{pc}$.

To get some idea on the completeness of the sample, one can plot the data from Fig. 2 as a cumulative distribution. This distribution turns out to be similar to the same distribution derived from all HIPPARCOS stars out to a distance of about $30 \pm 5 \mathrm{pc}$ for our complete sample. Therefore we conclude that our sample is complete out to that distance. Since our sample mostly consists of G stars, we conclude that for these we are limited by the effective X-ray horizon and not the flux limit of the HIPPARCOS catalogue.

\section{Spectroscopic observations and data reduction}

Spectroscopic follow-up observations were carried out at three different observatories: ESO (La Silla, Chile), KPNO (Kitt Peak, USA), and DSAZ (Calar Alto, Spain). At ESO, the $1.52 \mathrm{~m}$ ESO telescope with the FEROS echelle spectrograph ( $R=48000,3550-9210 \AA$ ) was used. Observations at KPNO were performed using the $0.9 \mathrm{~m}$ Coudé Feed telescope with the Coudé spectrograph (camera 5, grating A, Ford $3 \mathrm{~K} \times 1 \mathrm{~K} \mathrm{CCD}$, $R=25000,6415-6730 \AA)$. At the DSAZ, we used the FOCES echelle spectrograph $(R=40000,3730-9550 \AA)$ on the $2.2 \mathrm{~m}$ telescope. The observing dates are summarized in Table 1 . In total, we were able to observe 748 out of the 754 candidate stars in our sample. The remaining 6 stars (HD 4635, HD 4741, HD 7924, HD 8049, HD 133002, and HD 209943) could not be observed because of bad weather.

In addition to our target stars, two radial velocity standard stars were usually observed each night. To determine rotational velocities, on each run several stars with known rotational velocity were observed to be used as calibrators. 
Table 1. Observing runs for this project. (*) Completely lost because of bad weather.

\begin{tabular}{ccl}
\hline \hline \multicolumn{1}{c}{ Date } & Observatory \\
\hline May 26-Apr. 4 & 1999 & KPNO \\
Oct. 3-Oct. 10 & 1999 & KPNO \\
Mar. 27-Apr. 1 & 1999 & ESO \\
Oct. 27-Oct. 30 & 2000 & ESO \\
Apr. 20-Apr. 23 & 2001 & ESO \\
Jul. 25-Jul. 27 & 2000 & DSAZ \\
Feb. 28-Mar. 1 & 2001 & DSAZ $(*)$ \\
Jan. 28-Jan. 30 & 2002 & DSAZ \\
Apr. 4 & 2002 & ESO \\
\hline
\end{tabular}

Data reduction of the KPNO and DSAZ observations, i.e. bias subtraction, flatfielding, extraction of the spectra, and wavelength calibration, was performed using standard IRAF tasks. The ESO data were reduced online during the observations using the data reduction pipeline provided by the FEROS team.

For the determination of radial velocities we first compared all spectra of radial velocity standard stars to each other for each individual observing run in order to check for zero-point shifts between different nights. Since we did not detect such an effect, the spectrum of each star was cross-correlated with all spectra of radial velocity standards for that run, and the results averaged. For the cross-correlation, the IRAF task rvsao was used. The resulting errors of the radial velocities are typically about $2-3 \mathrm{~km} \mathrm{~s}^{-1}$.

\section{Results}

The presence of strong $\mathrm{Li}$ I absorption is the prime indicator of youth in low-mass stars, since lithium is burned by nuclear reactions at the bottom of the convective zone, and thus is depleted already during the pre-main sequence evolution. In analyzing our spectra, we have therefore focused our attention on the $\mathrm{Li}$ equivalent width $W_{\mathrm{LiI}}$.

According to the observed $W_{\mathrm{Li}}$, we have subdivided our sample into several groups, as detailed below, and we present results on the X-ray activity, kinematics, and spatial positions for individual subgroups.

\subsection{Lithium}

In Fig. 3, we plot the lithium equivalent width $W_{\mathrm{Li} \text { I }}$ vs. the effective temperature (as derived from $(B-V)_{J}$ ) for our sample stars with significant lithium detections. Also, we show the upper and lower envelope of Pleiades stars (data from Soderblom et al. 1993).

Our main criterium for confirming the youth of a star is that the star should have a positive $\Delta_{\mathrm{Li}}$, where $\Delta_{\mathrm{Li}}$ is defined as the star's equivalent width $W_{\mathrm{Li} \text { I }}$ diminished by the upper limit of $W_{\mathrm{Li} \text { I }}$ for stars in the Pleiades open cluster at the same effective temperature $T_{\text {eff }}$.
Table 2. Breakdown of the sample studied by us (total $=748$ stars).

\begin{tabular}{lrrr}
\hline \hline Sample & $N$ & Percentage & $\log \left(L_{\mathrm{x}} / L_{\mathrm{bol}}\right)$ \\
\hline "ZAMS sample" & 10 & 1.3 & $-3.49 \pm 0.07$ \\
"Pl sample" & 70 & 9.2 & $-3.91 \pm 0.07$ \\
Li below Pleiades & 286 & 38.4 & $-4.62 \pm 0.03$ \\
no Li & 392 & 57.4 & $-4.65 \pm 0.03$ \\
\hline
\end{tabular}

Lithium equivalent widths have been determined using the IRAF task splot. The error in the measured values of $W_{\mathrm{Li}}$ is primarily due to uncertainties in the continuum location. From repeated measurements we estimate it to about $15 \mathrm{~m} \AA$. The $\mathrm{Li}$ line is often blended with a weak nearby Fe I line at $\lambda 6707.44$. To account for this line, the correction prescribed by Favata et al. (1993) has been used. Judging from Fig. 1 in Favata et al. (1993), this introduces an additional statistical error of the order of 5-10 m

The statistical properties of the lithium criterium have been studied by Wichmann (2000) using the distribution functions of $\Delta_{\mathrm{Li}}$ f for several open clusters of different age. It was shown that this criterium has a very low error of the second kind (old stars erroneously classified as young). Because in the field old stars outnumber young ones by a large factor, this is a neccessary property of a useful criterium. On the other hand, the error of the first kind (young stars erroneously classified as old) is about 65 per cent, i.e. only about 35 per cent of young stars in the field can be found in this way.

According to our defined criterium, ten stars of our sample (see Fig. 3) can be classified as objects younger than the Pleiades. All of them are G-type stars, which we regard as an artefact of the bias towards G-type stars in our candidate sample. The spectra of these ten stars, in the region from $6700 \AA$ to $6720 \AA$, are shown in Fig. 8 .

It is worthwhile noting that the count statistics of the Pleiades sample, which is used to define our youth criterium, also is best for G-type stars, i.e. the upper envelope of the Pleiades $W_{\mathrm{Li}}$ is best defined in this region. Thus from a statistical perspective the criterium is much more robust for G-type stars than e.g. for K-type stars, where the upper envelope may change significantly if only a single star is removed from the Pleiades sample.

The breakdown of our sample is given in Table 2. In addition to the ten extremely Li-rich stars, there are some 356 more stars (i.e. about 48 per cent of our sample) with Li detections. Most of these stars are below the lower envelope of the Pleiades open cluster, and are probably several hundred Myr old. There are, however, some 70 stars in between the upper and lower Pleiades envelope, which thus might have ages similar to the Pleiades. (In fixing the Pleiades lower envelope, we have excluded two outlying G-type stars with very low $W_{\mathrm{LiI}}$. The inclusion of these two stars would primarily increase the pollution with older stars.)

We define a "ZAMS sample" comprising the 10 stars above the Pleiades upper envelope, and a "Pl sample" comprising the 70 stars in between the Pleiades upper and lower envelope. We will focus our attention primarily on these two groups of 


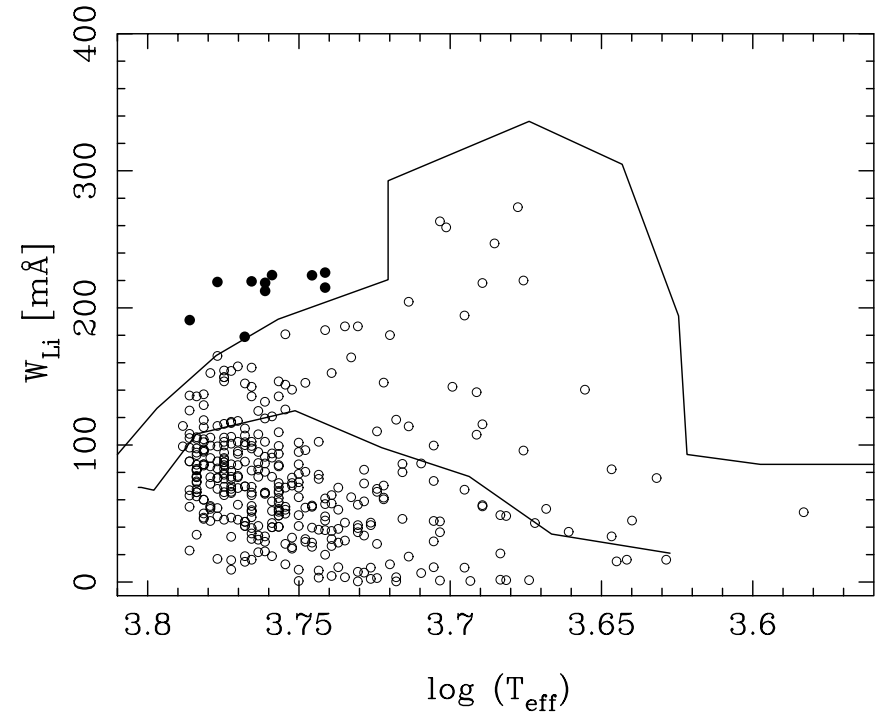

Fig. 3. $W_{\mathrm{Li}}$ plotted as function of $\log \left(T_{\text {eff }}\right)$. The two lines show the upper and lower limits for the Pleiades open cluster, respectively. Filled circles indicate stars of our survey located above the Pleiades upper limit (i.e. with $\Delta_{\mathrm{Li}}>0$ ), open circles stars with some lithium, but below the Pleiades upper limit $\left(\Delta_{\mathrm{Li}} \leq 0\right)$.

objects, which presumably represent the youngest stars within our sample, and we will try to characterize them in more detail.

\subsection{Location in the Herzsprung-Russel diagram}

In Fig. 4, we show the location of the "ZAMS sample" and the "Pl sample" in the Herzsprung-Russel diagram, together with evolutionary tracks from Siess et al. (2000).

With respect to the "ZAMS sample", most of the stars are right on the ZAMS. The star far below (HD 36869) has no HIPPARCOS parallax, only one from TYCHO, with a rather large error (28.6 \pm 7.1 mas). The star above the ZAMS (HD 105070) has a HIPPARCOS parallax of $9.78 \pm 1.16$ mas, thus the placement above the ZAMS has a $2 \sigma$ confidence level only.

For the "Pl sample", again most of the stars are on the ZAMS. There are three stars significantly below the ZAMS, all of which have only TYCHO parallaxes: BD+60 1417 (56.6 \pm 12.1 mas), BD+86 184 (28.9 \pm 7.7 mas), and HD 29623 (26.2 \pm 7.2 mas). With respect to the three stars that are located high above the ZAMS, one (HD 99409) has only a TYCHO parallax (29.4 \pm 7.8 mas). The other two are HD 224085 (II Peg) and HD 155555 (V824 Ara), which are both RS CVn binaries, i.e. evolved objects.

It is well known that RS CVn binaries have relatively high lithium surface abundances, although the reasons are not entirely clear (cf. Randich et al. 1994). These authors favour the hypothesis that the stars have evolved from massive progenitors that have suffered little or no Li depletion on the MS (because they lack an outer convection zone).

We conclude that neither in the "ZAMS sample" nor in the "Pl sample" are there objects that can unambiguously be identified as pre-main sequence stars based on their position in the $\mathrm{H}-\mathrm{R}$ diagram. This is not really surprising, because our

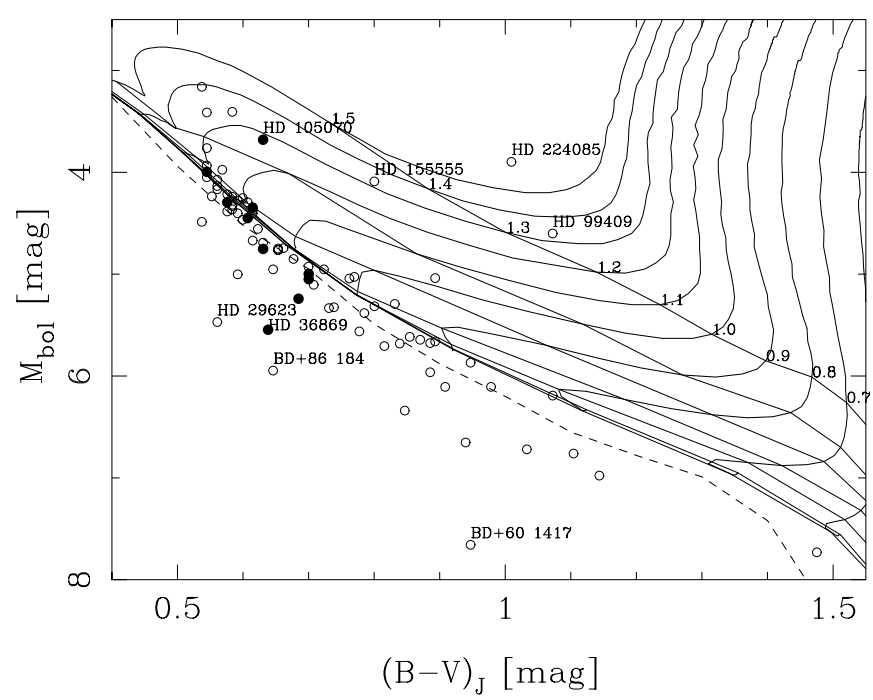

Fig. 4. H-R diagram of the youngest stars within our sample. Filled circles: stars with $\Delta_{\mathrm{LiI}}>0$, open circles: stars with $W_{\mathrm{Li}}$ in between the Pleiades lower and upper limit. Also plotted are evolutionary tracks from Siess et al. (2000) (left to right: $1.5-0.5 M_{\odot}$, in steps of $0.1 M_{\odot}$ ), with isochrones for $10, \mathbf{2 0}, \mathbf{3 0}, \mathbf{4 0}, 50$, and $100 \mathrm{Myr}$. The dashed line is the empirical ZAMS from Schmidt-Kahler (1982).

candidate sample is biased towards G-type stars, which have rather short pre-main sequence evolution times compared to Kor M-type stars.

\subsection{X-ray activity}

The X-ray activity, as measured by $\log \left(L_{\mathrm{x}} / L_{\text {bol }}\right)$, shows a very significant decline from the "ZAMS sample" to the "Pl sample", and a further, also very significant decline from the "Pl sample" to stars with $W_{\mathrm{Li}}$ below the Pleiades lower limit (see Table 2).

The difference between stars with and without Li detection below the Pleiades lower limit is quite small, and not statistically significant.

The clear trend of decreasing activity within our (sub-)samples shows that our method of determining ages by comparing $W_{\mathrm{Li} \text { I }}$ against the distribution observed in an open cluster (here: the Pleiades) is essentially valid and yields useful results.

If we restrict our analysis to the range in $B-V$ covered by the "ZAMS sample" $(0.54 \leq B-V \leq 0.70), \log \left(L_{\mathrm{x}} / L_{\mathrm{bol}}\right) \mathrm{de}-$ creases as $-4.23 \pm 0.04,-4.70 \pm 0.03,-4.68 \pm 0.08$ from the "Pl sample" to stars without lithium detection, i.e. our conclusions remain unaffected.

When comparing X-ray luminosities, i.e. $\log \left(L_{\mathrm{x}} / \mathrm{erg} \mathrm{cm}^{-2}\right)$ instead of X-ray activity, there is no clear trend with lithium if the full samples are used. The observed X-ray luminosities are $29.54 \pm 0.21$ ("ZAMS sample"), $29.61 \pm 0.10$ ("Pl sample"), $28.93 \pm 0.04$ (Li below Pleiades), and 29.19 \pm 0.04 (no Li detection). The reason is probably that active stars have a higher surface flux, but X-ray luminosity measures the product of surface and surface flux, thus giving a large weight to relatively massive (hence large), but less active stars.

If we again restrict our analysis to the $B-V$ range of the "ZAMS sample", the X-ray luminosities are $29.54 \pm 0.21$, 


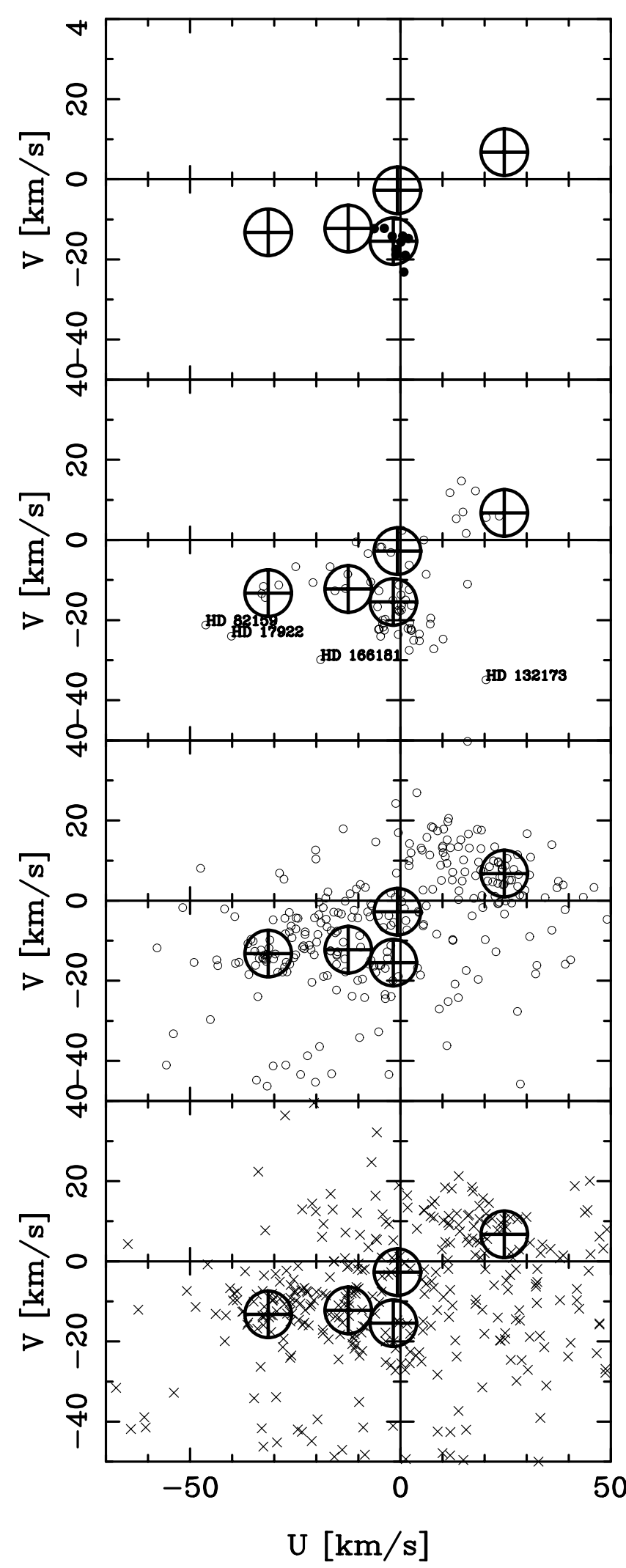

Fig. 5. Plot of $U$ vs. $V$ velocities. Velocities are relative to the local standard of rest (LSR). From top to bottom: stars with $\Delta_{\mathrm{LiI}}>0$, stars with $W_{\mathrm{Li}}$ in between the Pleiades lower and upper limit, stars with $W_{\text {Li I }}$ below the Pleiades lower limit, and stars without Li detection. Also marked are the locations of five superclusters (SCs) and moving groups (MGs) (left to right): Hyades MG, IC 2391 SC, Pleiades MG, Castor MG, Ursa Mayor MG.
$29.18 \pm 0.11,28.86 \pm 0.05$, and $28.88 \pm 0.08$, respectively. We therefore conclude that the lack of correlation of $\log \left(L_{\mathrm{x}}\right)$ with lithium in the full sample is due to the mass dependency discussed above.

\subsection{Kinematics}

Using the radial velocities determined from our spectra, as well as proper motions from HIPPARCOS (or TYCHO if not available), we have computed the galactic UVW velocities for all observed stars in our sample. All velocities were transformed into the local system of rest (LSR) using the solar motion $\left(U_{\odot}=10.0 \mathrm{~km} \mathrm{~s}^{-1}, V_{\odot}=5.25 \mathrm{~km} \mathrm{~s}^{-1}, W_{\odot}=7.17 \mathrm{~km} \mathrm{~s}^{-1}\right) \mathrm{de}-$ termined by Dehnen \& Binney (1998). We use the right-handed coordinate system ( $U$ towards the galactic centre, $V$ in the direction of the galactic rotation, and $W$ towards the north galactic pole).

In Fig. 5, we show a sequence of scatter plots of $U$ vs. $V$ velocities in the local standard of rest (LSR) for the following four samples: (top) our "ZAMS" sample, (2) our "Pl" sample, (3) stars with $W_{\mathrm{Li} \text { I }}$ below the Pleiades lower limit, and (bottom) stars without lithium detection.

Looking at the distribution of all stars in these diagrams, obviously the stars with Li detections are not distributed randomly, but rather cluster in some preferred regions in velocity space. In the terminology of Eggen (1994), who pioneered the study of these stellar kinematic groups, one defines a "supercluster" as a spatially extended, gravitationally unbound system of stars with a common velocity field, and a "moving group" (MG) as that part of such a supercluster that is within the solar neighbourhood (and spread all over the sky). In our $U-V$ diagrams, the most prominent MGs (the Local Association, also called the Pleiades MG, the Ursa Major MG, and the Hyades MG) are clearly visible. We also can observe a very clear shift in the clustering pattern with $\Delta_{\mathrm{Li} \mathrm{I}}$, i.e. the quantity we use to estimate ages. This shift demonstrates clearly that our method of measuring ages, using the offset of $W_{\mathrm{Li}}$ with respect to some open cluster, is confirmed by the kinematics of the samples thus obtained.

The ten stars of the "ZAMS sample" form a very tight kinematic group (which is also true if the $Z$ velocities are considered). The location in velocity space of this kinematic group is consistent with the Local Association (Pleiades MG).

The stars of the "Pl group" mostly cluster in the same region of the $U-V$ diagram. There are only few stars in the region of the (older) Ursa Major and Hyades MGs. There are also about four stars which are scattered somewhat apart from the rest. One (HD $166181=\mathrm{V} 815$ Her) is an RS CVs, one other (HD 132173) is a G0 star that has received little attention so far, while the other two (HD 17922 and HD 82159) are known as active young stars.

Stars with Li detection, but below the Pleiades lower limit, also show a distinct clustering pattern, but now the region of the Local Association is sparsely populated, while the regions of the (older) Ursa Major and Hyades MGs are well populated. This indicates clearly that this is a population older than the "Pl group". 


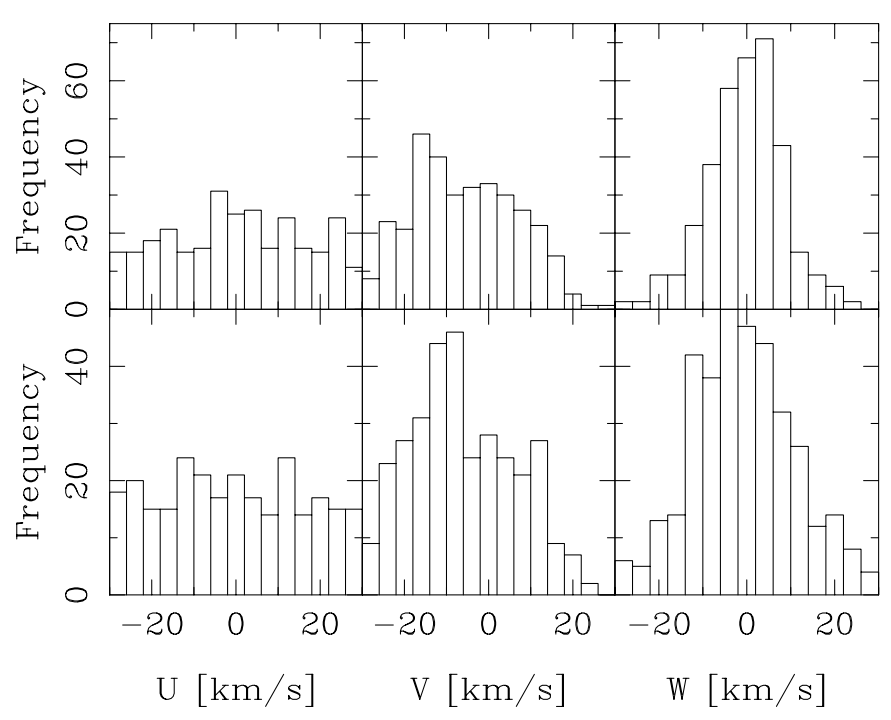

Fig. 6. Histograms of $U, V$, and $W$ velocities (in the LSR). Upper panel: stars with lithium detected, lower panel: stars without lithium detection.

Finally, the stars without Li detection show a much more scattered pattern, where the MGs are not as dominant as in the other samples. Presumably this sample includes many older disk stars, which are not associated with the MGs discussed before.

We can also use the $W$ velocities (perpendicular to the galactic plane) in order to confirm that those stars where lithium has been detected are on average younger than those without lithium detection. In Fig. 6 we show the histograms of $U, V$, and $W$ velocities for all stars with lithium detection (upper panel) and all stars without lithium detection (lower panel).

When comparing the distributions of the $U$ and $V$ velocities of the two samples, a two-sample KS test results in probabilities of $p=0.07$ and $p=0.09$ for the null hypothesis that both samples - stars with and without lithium - are drawn from the same parent population. Thus there is no significant difference for the $U$ and $V$ velocities. However, for the $W$ velocities the KS test yields a very low probability $(p=0.006)$ of the null hypothesis that both samples are drawn from the same parent population.

This effect can be seen in in Fig. 6. Obviously the $W$ velocities (i.e. perpendicular to the galactic plane) exhibit a markedly narrower distribution for the stars with lithium detection. We find $\bar{W}=-0.3, \sigma_{W}=9.7$ for the stars with lithium detection, and $\bar{W}=0.1, \sigma_{W}=15.9$ for those without. While the mean values are not significantly different, according to the F-test the difference in the dispersions is significant at the $p=10^{-5}$ level (i.e. there is a chance probability of less than $10^{-5}$ for a difference that large if the parent populations were the same).

It is well known that $\sigma_{W}$ increases with age, and according to a recent analysis of Haywood et al. (1997), the dispersions derived for our samples would correspond to typical ages of about $\leq 1$ Gyr (stars with lithium) and 3-5 Gyr (stars without lithium).

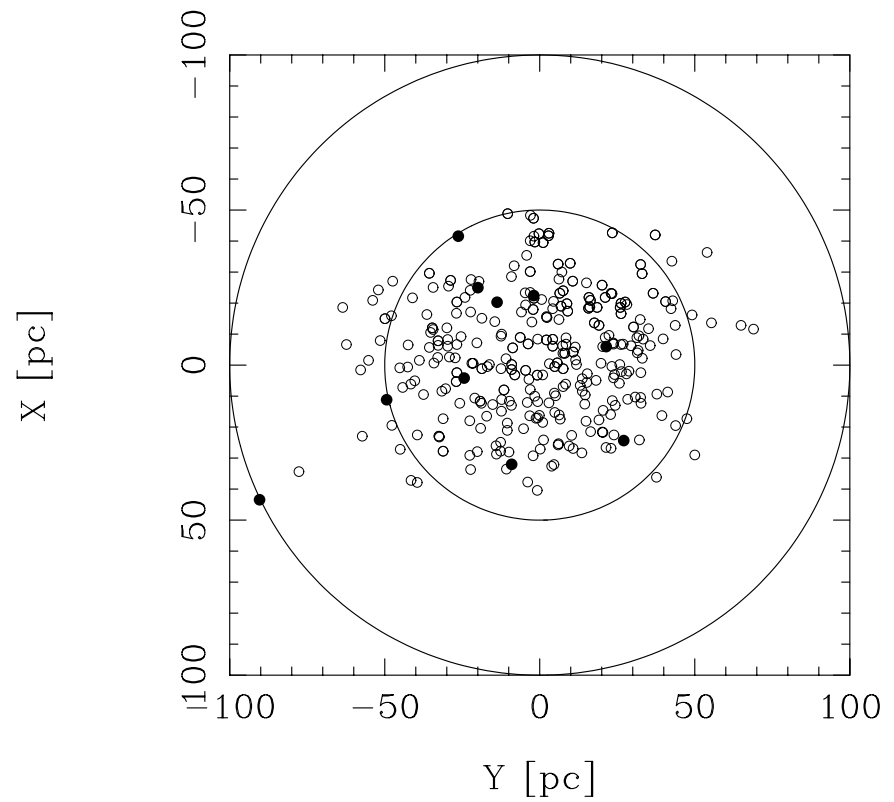

Fig. 7. Positions of stars with Li detected in absorption, projected on the galactic plane. The Sun is at the centre, and distances of 50 and $100 \mathrm{pc}$ are marked by circles. Filled circles: stars of the "ZAMS sample", open circles: other stars with Li absorption. (Galactic centre is towards bottom, galactic rotation towards right).

The ten stars of our "ZAMS sample" have $\sigma_{W}=3.1$, which would place them in the " $<0.15$ Gyr" age bin.

\subsection{Positions}

In Fig. 7, we show the positions of our sample stars projected onto the galactic plane. Clearly, although the stars of our "ZAMS sample" form a very tight kinematic group, their positions appear to be randomly distributed.

For a statistical analysis of the positions of our sample stars we need to take into account that our sample is biased with respect to distance, because both the TYCHO catalogue and the RASS are magnitude limited. Since the magnitude limits of both catalogues apply to completely different wavelength bands, it would be difficult, if not impossible, to treat the resulting bias properly in a statistical analysis. We therefore did not study the $3 \mathrm{~d}$ spatial distribution of our sample, but rather the $2 \mathrm{~d}$ distribution on the sphere. This $2 \mathrm{~d}$ distribution does not suffer from spatial bias, since our survey covered the whole sky.

Using the nearest neighbour distance (NND) statistics for the distribution on the sphere, we find that there are no significant differences between any of our subgroups ("ZAMS sample", "Pl sample", stars with and without lithium detection). However, all except the "ZAMS sample" show a significant overabundance of short distances, indicating some nonrandomness in the spatial distribution. We thus excluded the "ZAMS sample" from further analysis.

Following Fisher et al. (1987), we use the orientation matrix (inertia matrix) and its eigenvectors and eigenvalues to analyze the distribution of the other three subsamples on the sphere in more detail. Based on the value of the smallest normalized eigenvalue $\left(\bar{\tau}_{1}\right)$, we can reject the hypothesis of 

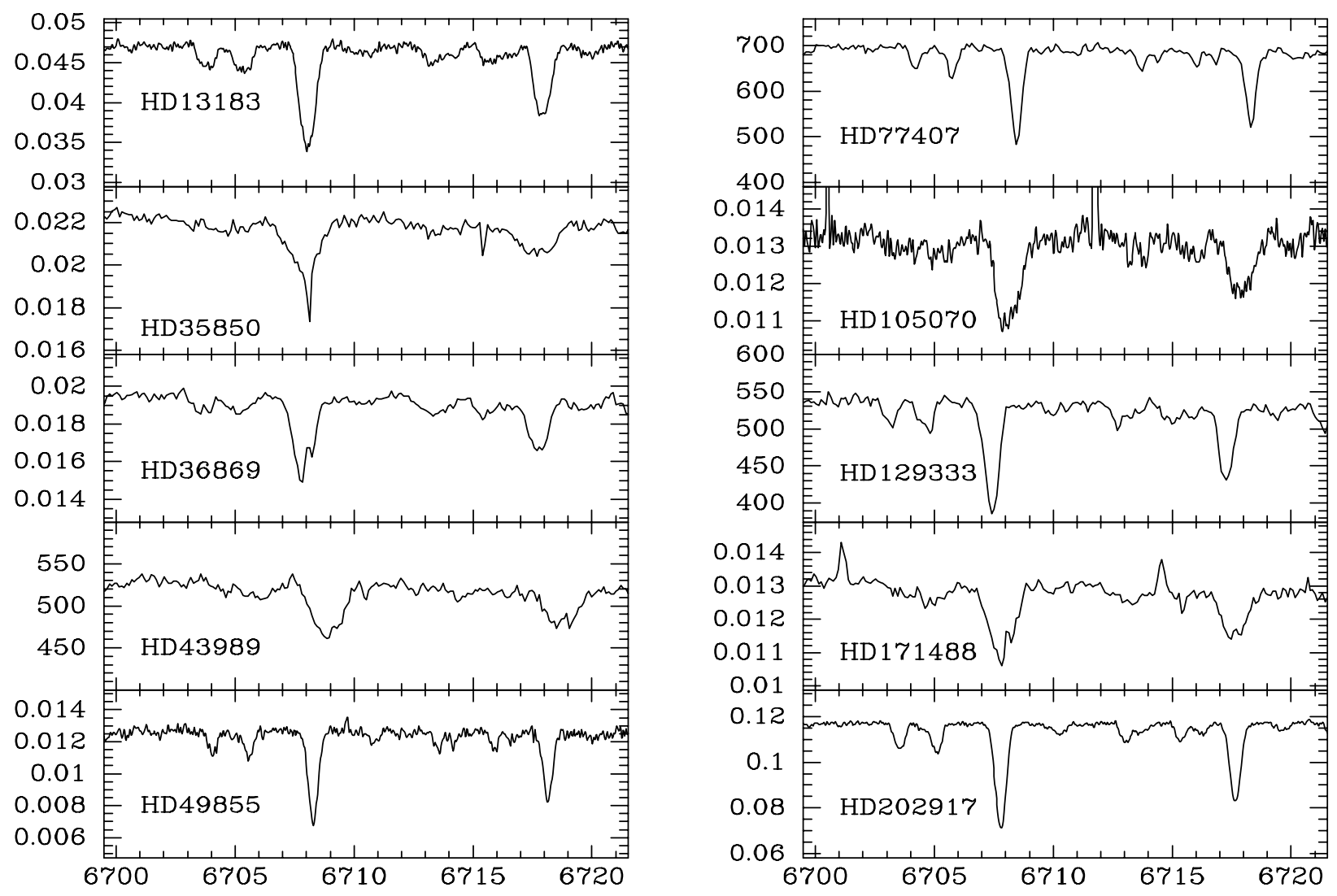

Fig. 8. Spectra of those stars classified as younger than Pleiades. No correction for heliocentric velocity has been applied.

uniformity against a girdle (or "belt") alternative at the one per cent level for all three of them. On the other hand, the empirical strength parameter $\zeta=\log \tau_{3} / \tau_{1}$ is always close to one, indicating that the deviation from uniformity is rather mild.

The eigenvector corresponding to the smallest eigenvalue, i.e. the polar axis in case of a girdle-like (belt-like) distribution, is well aligned with the galactic $Z$-direction (within $7 \mathrm{deg}$ ) in all three subsamples. We therefore conclude that the "Pl sample", as well as the samples of stars with and without lithium detection, are concentrated towards the galactic plane. While it would be interesting to compare the scale heights of these samples, we cannot do so since the scale height of the younger stars is artificially inflated with respect to the older ones because of the magnitude bias in the RASS.

The Gould Belt is inclined some 20 deg with respect to the galactic plane. Thus in the three subsamples where a detailed analysis is possible, we do not find evidence for the presence of a population related to the Gould Belt. However, we would expect such evidence only for the youngest of our subsamples, which unfortunately has too few stars for a meaningful statistical analysis.

\section{The ZAMS sample}

\subsection{Individual stars}

In the following section we discuss the individual stars of our "ZAMS" sample, the presumably youngest objects in the solar neighbourhood within our candidate sample. Some of these stars (see notes below) are flagged as "unsolved variable" in the HIPPARCOS catalogue. This indicates that there is some variability for a reason that could not be determined by the HIPPARCOS data reduction (e.g. because of poor phase coverage in the case of periodic variability). We presume that these stars are variable because of starspots, as this is the most likely reason for variability in active young stars. Based on the crosscorrelation with radial velocity standard stars (see Sect. 3), as well as on visual inspection of the spectra, we do not see indications of binarity in the spectra. The occasional "double-peaks" in the lines (e.g. in HD 36869, HD 43989, and HD 171486) are consistent with the noise level, and do not show up in other lines or in the cross-correlation function.

HD 13183: Torres et al. (2000) regard this G5V star as a member of their proposed Horologium association. Cutispoto et al. (2002) identify the star as an SB1 binary and list $W_{\mathrm{Li}}=$ $224 \mathrm{m \AA}$ and $v \sin i=22.5 \mathrm{~km} \mathrm{~s}^{-1}$.

HD 35850 (HR 1817): this is a well-known active star classified as F8V in Simbad. It has been studied in detail in the EUV and X-ray wavelengths (Gagne et al. 1999; Tagliaferri et al. 1997). Tagliaferri et al. (1994) have determined a lithium abundance of $\log N(\mathrm{Li})=3.2$ from their optical spectroscopy, but do not list the value of $W_{\mathrm{Li}}$ determined by them. There is no indication of multiplicity. The HIPPARCOS solution is flagged for "unsolved variability", which could indicate variability due to starspots, as expected for an highly active star. 
HD 36869: Montes et al. (2001) classify HD 36869 as a member of the Local Association. It is a G3V star, and detected in the EUV (Pye et al. 1995). Cutispoto et al. (2002) list $W_{\mathrm{LiI}}=260 \mathrm{m \AA}$ and $v \sin i=30 \mathrm{~km} \mathrm{~s}^{-1}$ for this star. There is no HIPPARCOS parallax available. The TYCHO parallax $(28.6 \pm 7.1$ mas) would place the star below the ZAMS.

HD 43989 (V1358 Ori): HD 43989 is detected in the EUV (Pounds et al. 1993). Photometry by Cutispoto et al. (1995) shows that the star has a rotational period of $3.63 \pm 0.13 \mathrm{~d}$. Osten \& Saar (1998) have used template fitting on optical spectra to determine the parameters of this star. Their best fits are for a single G0IV subgiant with $T_{\text {eff }}=5900 \mathrm{~K}, v \sin i=42 \mathrm{~km} \mathrm{~s}^{-1}$, or a double G0IV+G0IV subgiant with the same $T_{\text {eff }}$, but lower $v \sin i\left(25 \mathrm{~km} \mathrm{~s}^{-1}\right)$. From our spectrum, we determine a $v \sin i=40 \mathrm{~km} \mathrm{~s}^{-1}$, indicating that either the star is single, or that by chance, we have observed it at a similar orbital phase as Osten \& Saar. We regard the first alternative as the more likely one. The HIPPARCOS solution is flagged for "unsolved variability". Based on the HIPPARCOS distance, the star is located on the ZAMS.

HD 49855: apparently, this G6V star has not been studied in any detail so far (the only reference in SIMBAD is for a $u v b y$ survey by Olsen 1994). The HIPPARCOS data are flagged for unsolved variability.

HD 77407: this star is detected as a radio source with a flux of $1.67 \mathrm{mJy}$ at $20 \mathrm{~cm}$ (Helfand et al. 1999), and also as an EUV source (Mason et al. 1995). Montes et al. (2001) identify HD 77407 as a member of the Local Association, based on kinematic criteria, and determine $W_{\mathrm{Li} I}=170 \mathrm{~m} \AA$, in agreement with our data $(183 \mathrm{~m} \AA)$. There are no spectroscopic indications of multiplicity, and no variability observed by HIPPARCOS.

HD 105070: HD 105070 is a G1V star that is considered as a member of the Lower Centaurus Crux OB association by De Zeeuw et al. (1999), based on HIPPARCOS positions, proper motions, and parallaxes. However, the astrometric radial velocity of this association $\left(5.95 \pm 0.53 \mathrm{~km} \mathrm{~s}^{-1}\right)$, as determined from proper motions and parallaxes by Madsen et al. (2002), seems to be at variance with the spectroscopic radial velocity of HD 105070 measured by us $\left(11.9 \mathrm{~km} \mathrm{~s}^{-1}\right)$. The HIPPARCOS parallax of this star is not very accurate $(9.78 \pm 1.16$ mas $)$ and places the star above the ZAMS.

HD 129333 (EK Dra): HD 129333 is a well-known BY Draconis variable of spectral type F8, with a photometric period of 2.605 d (Strassmeier et al. 1997). Montes et al. (2001) classify it as a member of the Local Association and determine $W_{\mathrm{Li} \text { I }}=200 \mathrm{~m} \AA$, in good agreement with our data. HD 129333 is detected in the EUV (Pounds et al. 1993).
HD 171488 (V889 Her): HD 171488 is a G2V star that is detected as an EUV source (Pounds et al. 1993). Based on kinematic criteria, Montes et al. (2001) regard it as a member of the Local Association. Henry et al. (1995) have measured a photometric period of $1.338 \mathrm{~d}$ and $v \sin i=33 \mathrm{~km} \mathrm{~s}^{-1}$ for this star, while Cutispoto et al. (2002) determine $v \sin i=45 \mathrm{~km} \mathrm{~s}^{-1}$, as well as $W_{\mathrm{Li}}=220 \mathrm{~m} \AA$ (in perfect agreement with our measurement). Both Osten \& Saar (1998) and Fekel (1997) list $v \sin i=38 \mathrm{~km} \mathrm{~s}^{-1}$ for HD 171488 . The star is flagged as unsolved variable in the HIPPARCOS database, which is likely due to starspots.

HD 202917: HD 202917 is a G5V star detected as a faint EUVE source (Lampton et al. 1997). It is regarded as a member of the local association by Montes et al. (2001), and as a member of their proposed Tucana association by Zuckerman \& Webb (2000). Cutispoto et al. (2002) list $W_{\mathrm{LiI}}=225 \mathrm{~m} \AA$ and $v \sin i=14 \mathrm{~km} \mathrm{~s}^{-1}$ for this star. The star is flagged as unsolved variable in the HIPPARCOS database.

\subsection{The missing candidates}

There are a few well-known active (and presumably young) stars in the solar neighbourhood that one might expect to be members of our "ZAMS sample", yet that are missing in our survey, or at least in our list of the youngest stars (i.e. the "ZAMS sample"). Here we discuss briefly the most prominent stars in this category, and the reasons why they are missing.

UV Cet: this is a well known, very active flare star of spectral type M5.5e. Due to its faintness $\left(m_{V}=12.5\right)$ it is not included in the TYCHO catalogue, hence also not in our survey.

GJ 182 (V1005 Ori): this dM0.5 star $\left(m_{V}=10.1\right)$ is among the coronally most active stars in the solar neighbourhood (Sterzik \& Schmitt 1997). It has dropped out of the candidate list during the initial selection because of the large relative error of its TYCHO parallax, upon which the selection was based.

HD 36705 (AB Dor): according to the HIPPARCOS photometry, this very active young $\mathrm{K} 0 \mathrm{star}\left(m_{V}=6.93\right)$ is located on the ZAMS (Wichmann et al. 1998). The star is included in our survey, and we measured $W_{\mathrm{LiI}}=282 \mathrm{~m} \AA$, in agreement with Hussain et al. (1997). With this value of $W_{\mathrm{Li}}$, the star is not a bona-fide ZAMS star according to our lithium criterium (it is $35 \mathrm{~m} \AA$ below the Pleiades upper envelope).

We would like to remind the reader that because of the large intrinsic spread in $W_{\mathrm{Li}}$, our criterium has a large error of the first kind, i.e. many ZAMS stars will not be recognized by it. On the other hand, the UVW velocities of $A B$ Dor are 2.5, -17.1 , and $-12.0 \mathrm{~km} \mathrm{~s}^{-1}$ respectively. This would make $\mathrm{AB}$ Dor the star most outlying from the sample mean, if plotted as a member of the "ZAMS sample" in the top panel of Fig. 5. Thus, also the kinematics is somewhat discordant with the "ZAMS sample". 
Table 3. Spectroscopically observed stars (ZAMS sample). Listed are the designation of each star, the position (J2000.0), $W_{\mathrm{Li} I}^{\mathrm{m}}$ (mÅ, measured), $W_{\mathrm{Li} \text { I }}^{\mathrm{c}}\left(\mathrm{m} \AA\right.$, corrected for Fe I), $\Delta_{\mathrm{Li}}(\mathrm{m} \AA)$, radial velocitiy $V_{\text {rad }}\left(\mathrm{km} \mathrm{s}^{-1}\right)$, rotational velocity $v \sin i\left(\mathrm{~km} \mathrm{~s}^{-1}\right)$, galactic $U V W$ velocity components, the parallax used (mas), and $\log \left(L_{\mathrm{x}}\right)$. Errors for $V_{\text {rad }}$ are typically $2-3 \mathrm{~km} \mathrm{~s}^{-1}$, errors for $v \sin i$ about 10 per cent.

\begin{tabular}{lllllrrrrrrrr}
\hline \hline Designation & RA (2000.0) & Dec (2000.0) & $W_{\mathrm{LiI}}^{\mathrm{m}}$ & $W_{\mathrm{Li}}^{\mathrm{c}}$ & $\Delta_{\mathrm{LiI}}$ & $V_{\mathrm{rad}}$ & $v \sin i$ & $U$ & $V$ & $W$ & $\pi$ & $\log \left(L_{\mathrm{x}}\right)$ \\
\hline HD 13183 & 020717.98 & -531156.40 & 225 & 215 & 11 & 9.8 & 22 & 0.1 & -15.7 & 6.6 & 19.9 & 29.4 \\
HD 35850 & 052704.75 & -115402.88 & 191 & 191 & 43 & 23.6 & 50 & -3.8 & -12.2 & -3.0 & 37.3 & 30.6 \\
HD 36869 & 053409.14 & -151703.12 & 230 & 224 & 35 & 24.9 & 29 & -6.2 & -12.3 & -1.1 & 28.6 & 29.6 \\
HD 43989 & 061908.04 & -032620.04 & 221 & 219 & 53 & 21.1 & 40 & -2.0 & -14.2 & 1.7 & 20.1 & 29.3 \\
HD 49855 & 064346.32 & -715835.76 & 236 & 226 & 22 & 20.1 & 12 & -1.1 & -17.5 & 1.8 & 17.7 & 28.6 \\
HD 77407 & 090327.12 & +375029.04 & 183 & 179 & 1 & 5.0 & $<10$ & -1.1 & -18.8 & -0.2 & 33.2 & 29.9 \\
HD 105070 & 120547.52 & -510011.88 & 224 & 218 & 32 & 11.9 & 32 & 0.6 & -14.1 & 0.9 & 9.8 & 28.4 \\
HD 129333 & 143900.48 & +641730.12 & 218 & 212 & 26 & -20.5 & 18 & 0.8 & -23.1 & 1.7 & 29.5 & 30.0 \\
HD 171488 & 183420.16 & +184124.72 & 224 & 219 & 39 & -24.5 & 39 & 1.2 & -19.0 & 1.6 & 26.9 & 30.1 \\
HD 202917 & 212049.92 & -530202.40 & 233 & 224 & 23 & -1.4 & 13 & 1.9 & -14.7 & 6.7 & 21.8 & 29.5 \\
\hline
\end{tabular}

HD 197890 (Speedy Mic, BO Mic): this very active K0 star $\left(m_{V}=9.44\right)$ has dropped out of the initial selection because of its grossly incorrect TYCHO parallax that placed it way below the main sequence.

\subsection{Discussion}

One particularly striking result of our study is that there seems to be a group of very young and highly active stars in the solar neighbourhood that form a tight kinematic group, yet at the same time show a large dispersion in space. While most of the 10 stars that we propose for this group have already been individually studied by other authors, the strong similarities between these stars, in particular their extremely high levels of $W_{\mathrm{Li}}$, their small velocity dispersion, and their high degree of $\mathrm{X}$-ray activity have gone unnoticed so far.

The high $W_{\mathrm{Li}}$ indicates that these stars are almost certainly younger than the Pleiades. Another limit may be obtained from their velocity dispersions $\left(\sigma_{W}=2.5, \sigma_{V}=3.4, \sigma_{W}=3.1\right)$. The diffusion of stellar orbits, and the resulting increase in velocity dispersion, has been studied in some detail by Wielen (1977). Comparing our observed dispersions with his Table 2, we obtain an upper limit of 50-100 Myr. (This is an upper limit only because we do not know the initial velocity dispersion.)

We presume that these stars begun to disperse almost immediately after their birth, contrary to the many well-known long-lived open clusters like the Pleiades. As already discussed by Wielen (1971), these open clusters can account only for a small percentage of field stars. One can therefore expect that on average, newly formed clusters of stars will not become long-lived open clusters, but disperse rather quickly. The young kinematic group identified by us might represent such a case.

An alternative scenario would be ejection from a young cluster by three-body interaction. This is very unlikely given the small velocity dispersion exhibited by our ZAMS sample. The origin of these stars, i.e. their possible place(s) of formation, will be the subject of a forthcoming paper (Wichmann \& Schmitt, submitted).
With a normal IMF (cf. Kroupa et al. 1993), the observed number of 10 stars in the range F8-G5 corresponds to 19 F8-G9 stars, $51 \mathrm{~K}$ stars, and 1460 stars from M0 to the substellar limit. The total mass of the population, including high-mass stars, would be about $300 M_{\odot}$ (where stars earlier than F8 contribute some $15 M_{\odot}$ ). Clearly, because of the magnitude limit of the TYCHO catalogue and the RASS, we are missing most of the young field stars that belong to this population.

\section{Conclusions}

In the course of the survey presented here, we have found in the solar neighbourhood 10 G-type field stars in our sample with very similar kinematics that appear to be significantly younger than the Pleiades. While some of these stars are actually already known for their activity, and have been suspected to be young before, we think this is the first work to systematically identify such stars, and to show kinematic similarities between them.

If we restrict the completeness analysis of our sample from Sect. 2 to G stars only, we estimate that our sample is complete out to about $40 \pm 5 \mathrm{pc}$. 6 of our 10 young stars are within that radius, yielding a space density of about $2.2 \times 10^{-5}$ per cubic parsec, with an error of about a factor 2.

Furthermore, our analysis of the detection method itself allows us to provide some estimate of the error of the first kind, which is on the order of 65 per cent. Judging from this, there should be some 20 more young G-type stars in our sample that fail the lithium test because of the intrinsic spread in $\Delta W_{\mathrm{Li}}$.

We further find within our candidate sample some 70 stars with $\Delta W_{\mathrm{Li}}$ in the Pleiades range, and some 286 more stars with detectable lithium.

Our results clearly show that there are abundant young stars in the field. We are clearly sampling only a small part of this population. This is primarily caused by the magnitude limitation of the TYCHO catalogue, which causes a bias towards early-type stars.

One of the original motivations for starting this survey has been the Guillout et al. (1998) model of a "truncated disk" for the Gould Belt. Unfortunately, we can neither rule out nor 
Table 4. Spectroscopically observed stars (Pl sample). Listed are the designation of each star, the position (J2000.0), $W_{\mathrm{Li}}^{\mathrm{m}}$ (mÅ, measured), $W_{\mathrm{Li} \mathrm{I}}^{\mathrm{c}}(\mathrm{m} \AA$, corrected for $\mathrm{Fe} \mathrm{I}), \Delta_{\mathrm{Li} \mathrm{I}}(\mathrm{m} \AA)$, radial velocitiy $V_{\text {rad }}\left(\mathrm{km} \mathrm{s}^{-1}\right)$, rotational velocity $v \sin i\left(\mathrm{~km} \mathrm{~s}^{-1}\right)$, galactic $U V W$ velocity components, the parallax used (mas), and $\log \left(L_{\mathrm{x}}\right)$. Errors for $V_{\text {rad }}$ are typically $2-3 \mathrm{~km} \mathrm{~s}^{-1}$, errors for $v \sin i$ about 10 per cent.

\begin{tabular}{|c|c|c|c|c|c|c|c|c|c|c|c|c|}
\hline Designation & RA (2000.0) & $\operatorname{Dec}(2000.0)$ & $W_{\mathrm{Li} \mathrm{I}}^{\mathrm{m}}$ & $W_{\mathrm{Li} \mathrm{I}}^{\mathrm{c}}$ & $\Delta_{\mathrm{Li} \mathrm{I}}$ & $V_{\mathrm{rad}}$ & $v \sin i$ & $U$ & $\bar{V}$ & $\bar{W}$ & $\pi$ & $\log \left(L_{\mathrm{x}}\right)$ \\
\hline HD 105 & 000552.46 & -414510.44 & 157 & 154 & $\begin{array}{l}-18 \\
\text { n }\end{array}$ & 1.7 & 13 & 0.2 & -16.3 & 5.8 & 24.9 & 29.0 \\
\hline HD 377 & 000825.70 & +063700.48 & 161 & 156 & -24 & 1.3 & 15 & -4.4 & -1.8 & 3.3 & 25.1 & 29.1 \\
\hline HD 691 & 001122.30 & +302658.20 & 124 & 110 & -108 & -2.8 & $<10$ & -20.8 & -10.6 & 8.1 & 29.4 & 29.0 \\
\hline HD 987 & 001352.82 & -744117.52 & 199 & 186 & -26 & 9.3 & 6 & 1.2 & -15.0 & 5.5 & 22.9 & 29.5 \\
\hline HD 1405 & 001820.78 & +30 5723.76 & 298 & 273 & -59 & -12.0 & 23 & 7.2 & -19.5 & -4.8 & 45.7 & 30.5 \\
\hline HD 4944 & 005024.19 & -640404.08 & 138 & 137 & -20 & 10.7 & 28 & -7.1 & -10.5 & -3.3 & 18.4 & 29.1 \\
\hline HD 8558 & 012321.14 & -572850.52 & 194 & 184 & -20 & 9.2 & 12 & -0.5 & -17.5 & 6.3 & 20.3 & 29.3 \\
\hline HD 8813 & 012325.61 & -763642.12 & 133 & 126 & -68 & 13.0 & $<6$ & -1.1 & -19.1 & 5.2 & 20.7 & 28.4 \\
\hline HD 10195 & 014206.10 & +690509.60 & 154 & 152 & -9 & 11.4 & $<10$ & -28.9 & -11.2 & 14.9 & 21.1 & 28.8 \\
\hline HD 12039 & 015748.91 & -215405.04 & 188 & 181 & -13 & 6.6 & 17 & -0.6 & -16.3 & 5.0 & 23.6 & 29.1 \\
\hline HD 13482 & 021215.34 & +235730.96 & 160 & 145 & -74 & 0.3 & $<10$ & 1.8 & -22.1 & -5.6 & 31.0 & 29.4 \\
\hline HD 17925 & 025231.90 & -124609.48 & 215 & 194 & -122 & 18.2 & $<10$ & -5.4 & -16.6 & -2.1 & 96.3 & 31.1 \\
\hline HD 17922 & 025413.94 & +423520.40 & 99 & 99 & -44 & 24.6 & $<10$ & -40.1 & -24.0 & 5.6 & 19.4 & 28.8 \\
\hline HD 20367 & 031740.13 & +310737.92 & 114 & 113 & -44 & 6.6 & $<10$ & 11.8 & 11.8 & -7.9 & 36.9 & 29.6 \\
\hline HD 22705 & 033653.33 & -495728.80 & 157 & 154 & -14 & 11.8 & 17 & 0.1 & -13.6 & 8.3 & 24.0 & 29.2 \\
\hline HD 28344 & 042848.24 & +171707.80 & 121 & 117 & -57 & 39.4 & $<10$ & -32.1 & -14.4 & 6.2 & 21.1 & 28.6 \\
\hline HD 283750 & 043648.10 & +270757.00 & 65 & 33 & -275 & 39.4 & $<10$ & -32.5 & -11.6 & 4.8 & 56.0 & 31.0 \\
\hline HD 29623 & 043923.81 & -123147.64 & 119 & 118 & -39 & 4.6 & $<10$ & 13.3 & 5.3 & -5.1 & 26.2 & 28.5 \\
\hline HD 29697 & 044119.01 & +205407.56 & 78 & 45 & -241 & 0.5 & 12 & 15.6 & 1.6 & -13.9 & 74.1 & 31.1 \\
\hline HD 30311 & 044645.53 & +090103.00 & 130 & 129 & -28 & 44.4 & $<10$ & -33.0 & -13.4 & 0.8 & 26.3 & 29.1 \\
\hline HD 36705 & 052844.78 & -652656.04 & 284 & 267 & -36 & 37.4 & 53 & 2.1 & -27.5 & -12.3 & 66.9 & 31.5 \\
\hline HD 37572 & 053656.83 & -475752.92 & 282 & 263 & -46 & 32.1 & 9 & 2.6 & -22.6 & -7.4 & 41.9 & 30.4 \\
\hline HD 38397 & 054335.78 & -395524.96 & 167 & 165 & -1 & 22.9 & 16 & -1.7 & -15.0 & 1.9 & 19.2 & 28.9 \\
\hline HD 45270 & 062230.96 & -601307.68 & 161 & 157 & -17 & 30.9 & 17 & 2.5 & -22.1 & -6.9 & 42.6 & 30.1 \\
\hline HD 48189 & 063800.41 & -613200.96 & 149 & 145 & -33 & 30.1 & 17 & 2.2 & -20.6 & -8.9 & 46.1 & 30.7 \\
\hline HD 61005 & 073547.52 & -321214.76 & 176 & 164 & -47 & 22.0 & 8 & -12.5 & -8.5 & 2.8 & 28.9 & 29.2 \\
\hline HD 62850 & 074236.00 & -591752.08 & 147 & 142 & -38 & 17.1 & 15 & -15.5 & -12.7 & 4.9 & 30.1 & 29.2 \\
\hline HD 72687 & $0833 \quad 15.36$ & -295723.76 & 148 & 140 & -55 & 21.4 & $<6$ & -5.1 & -12.7 & 4.2 & 21.9 & 28.7 \\
\hline HD 72905 & 083911.76 & +650114.52 & 140 & 135 & -45 & -13.1 & 10 & 20.4 & 5.6 & -3.0 & 70.1 & 30.6 \\
\hline HD 75332 & 085032.16 & +331707.08 & 125 & 125 & -23 & 4.4 & 11 & 2.1 & -6.3 & 1.7 & 34.9 & 29.8 \\
\hline HD 78141 & 090718.00 & +225222.08 & 129 & 107 & -212 & -18.9 & 11 & 23.5 & 6.0 & -7.3 & 46.7 & 29.9 \\
\hline HD 82159 & 093036.00 & +103606.48 & 137 & 115 & -207 & 42.3 & 15 & -46.2 & -21.2 & 2.2 & 21.1 & 29.6 \\
\hline HD 82558 & 093225.68 & -111104.92 & 270 & 247 & -78 & 8.0 & 29 & -10.5 & -0.5 & -2.0 & 54.5 & 30.9 \\
\hline HD 82443 & 093243.92 & +265920.76 & 195 & 180 & -113 & 7.9 & $<10$ & 0.3 & -17.5 & 1.4 & 56.4 & 30.4 \\
\hline HD 90712 & 102747.76 & -342357.84 & 118 & 115 & -53 & 19.5 & 10 & -3.6 & -19.8 & -3.6 & 25.6 & 29.2 \\
\hline HD 90905 & 102942.24 & +012929.04 & 136 & 136 & -17 & 16.5 & 11 & -4.7 & -24.1 & -0.6 & 31.7 & 29.6 \\
\hline HD 92945 & 104328.32 & -290351.12 & 160 & 138 & -181 & 22.4 & $<6$ & -5.2 & -22.4 & 2.8 & 46.4 & 29.4 \\
\hline HD 93528 & 104731.20 & -222052.80 & 118 & 100 & -207 & 23.4 & $<6$ & -5.0 & -22.2 & 6.9 & 28.7 & 28.7 \\
\hline HD 96064 & 110441.52 & -041314.88 & 130 & 114 & -186 & 18.7 & 10 & -4.1 & -21.8 & 6.8 & 40.6 & 30.2 \\
\hline HD 99409 & 112558.32 & -401550.04 & 114 & 82 & -226 & 17.4 & 17 & 15.9 & -11.0 & 10.4 & 29.4 & 29.4 \\
\hline HD 104860 & 120433.84 & +662011.40 & 152 & 149 & -19 & -11.8 & 16 & 0.4 & 2.4 & -11.6 & 20.9 & 28.7 \\
\hline HD 105690 & 121006.48 & -491050.16 & 163 & 152 & -53 & 15.8 & 8 & -3.8 & -22.5 & -4.2 & 26.4 & 28.9 \\
\hline HD 107146 & 121906.48 & +163255.32 & 130 & 125 & -58 & 1.8 & $<10$ & -0.6 & -23.6 & 2.2 & 35.1 & 29.5 \\
\hline $\mathrm{BD}+601417$ & 124333.36 & +600053.28 & 121 & 96 & -238 & -10.0 & 11 & 6.1 & -8.6 & 1.2 & 56.6 & 29.2 \\
\hline $\mathrm{BD}+86184$ & 124402.88 & +852656.40 & 153 & 146 & -46 & -0.6 & $<9$ & -7.7 & -3.4 & 2.1 & 28.9 & 29.0 \\
\hline HD 113449 & 130349.68 & -050940.68 & 162 & 142 & -170 & 11.5 & 11 & 7.9 & -27.2 & 5.6 & 45.2 & 29.6 \\
\hline HD 113553 & 130516.80 & -505123.76 & 154 & 145 & -54 & 1.1 & 11 & -13.1 & -12.1 & 8.1 & 22.1 & 28.8 \\
\hline HD 126246 & 142405.76 & +111449.20 & 114 & 114 & -30 & 0.3 & $<10$ & 17.8 & 12.2 & 3.5 & 27.9 & 29.2 \\
\hline HD 130948 & 145015.84 & +235442.48 & 119 & 116 & -56 & -2.7 & $<10$ & 14.5 & 14.7 & -0.4 & 55.7 & 30.1 \\
\hline
\end{tabular}

confirm that the stars in our "ZAMS sample" are Gould Belt stars, as the sample is too small for a meaningful spatial analysis (the other subsamples are older than the Gould Belt).

The number of young stars found in our survey is in agreement with estimates (Wichmann 2000) for the expected fraction of about 0.02 for young stars in the field, in a scenario where stars would (approximately) instantaneously disperse into the field after formation, and under the assumption of a current star forming rate of 3.5-5 $M_{\odot} \mathrm{pc}^{-2} \mathrm{~Gy}^{-1}$ (see Rana 1991 and references therein). However, given the uncertainties 
Table 4. continued.

\begin{tabular}{llllrrrrrrrrr}
\hline \hline Designation & RA (2000.0) & Dec $(2000.0)$ & $W_{\mathrm{Li}}^{\mathrm{m}}$ & $W_{\mathrm{Li}}^{\mathrm{c}}$ & $\Delta_{\mathrm{Li}}$ & $V_{\mathrm{rad}}$ & $v \sin i$ & $U$ & $V$ & $W$ & $\log \left(L_{\mathrm{x}}\right)$ \\
\hline HD 131156 & 145123.04 & +190607.92 & 111 & 76 & -161 & 1.0 & $<10$ & 14.9 & 7.0 & 5.5 & 149.3 & 31.8 \\
HD 132173 & 145830.72 & -284233.48 & 136 & 136 & -12 & 26.7 & 8 & 20.4 & -34.9 & 13.1 & 20.4 & 28.5 \\
HD 135363 & 150756.64 & +761201.08 & 245 & 220 & -114 & -5.8 & 21 & -16.7 & -6.7 & -1.6 & 34.0 & 30.3 \\
HD 139813 & 152924.48 & +802700.00 & 134 & 119 & -177 & -15.1 & $<9$ & -4.2 & -20.0 & 4.8 & 46.0 & 30.3 \\
HD 152555 & 165408.16 & -042023.64 & 149 & 146 & -22 & -16.4 & 17 & 4.5 & -23.5 & -5.2 & 21.0 & 28.9 \\
HD 155555 & 171725.44 & -665702.52 & 221 & 205 & -95 & 6.2 & 31 & 2.1 & -12.4 & -2.2 & 31.8 & 30.7 \\
HD 166181 & 180815.84 & +294128.32 & 198 & 186 & -23 & -57.1 & 31 & -18.9 & -29.9 & -33.6 & 30.7 & 30.5 \\
HD 177596 & 190436.72 & +220525.44 & 152 & 149 & -19 & -25.1 & 31 & 10.1 & -24.7 & -1.7 & 11.6 & 27.7 \\
HD 181321 & 192129.76 & -345859.52 & 137 & 131 & -55 & -9.5 & 12 & -2.3 & -3.1 & 0.4 & 48.0 & 30.2 \\
HD 183414 & 193509.60 & -695830.72 & 142 & 135 & -56 & 3.0 & 9 & -4.2 & -20.8 & 6.3 & 28.2 & 29.2 \\
HD 187748 & 194815.36 & +592521.36 & 116 & 114 & -52 & -5.3 & $<10$ & -4.8 & -1.8 & 10.4 & 35.2 & 29.4 \\
HD 193464 & 202142.96 & -363636.00 & 108 & 108 & -40 & -28.7 & 13 & -24.9 & -6.7 & 4.8 & 16.5 & 28.6 \\
HD 197481 & 204509.36 & -312024.00 & 97 & 51 & -35 & -4.8 & 10 & -0.4 & -11.2 & -3.1 & 100.6 & 31.8 \\
HD 198767 & 204818.00 & +690830.12 & 120 & 117 & -55 & -28.9 & $<10$ & 4.5 & -25.2 & 0.6 & 27.8 & 28.7 \\
BD +22 4409 & 213101.68 & +232008.52 & 170 & 140 & -177 & -24.5 & 60 & 3.1 & -25.0 & -6.4 & 39.9 & 30.2 \\
BD +48 3686 & 222006.96 & +493011.88 & 278 & 259 & -52 & -19.8 & 20 & 2.6 & -16.3 & 5.4 & 42.4 & 30.3 \\
HD 217343 & 230019.20 & -260912.24 & 151 & 144 & -50 & 7.0 & 12 & 7.1 & -21.0 & -7.5 & 31.2 & 29.5 \\
HD 218866 & 231024.48 & +643148.00 & 105 & 105 & -43 & -4.0 & $<10$ & 5.6 & -0.0 & -6.1 & 26.1 & 29.1 \\
HD 220140 & 231925.92 & +790011.88 & 240 & 218 & -104 & -16.3 & 16 & -0.2 & -17.8 & 1.8 & 50.6 & 30.9 \\
HD 224085 & 235503.60 & +283800.96 & 65 & 37 & -286 & 11.7 & 22 & -97.0 & -32.7 & -18.6 & 23.6 & 30.8 \\
HD 224228 & 235610.56 & -390306.84 & 80 & 53 & -277 & 13.1 & $<6$ & 2.5 & -22.6 & -6.3 & 45.3 & 29.4 \\
\hline
\end{tabular}

involved in an extrapolation from 10 stars only, as well as in the determination of the current star forming rate, we cannot rule out the possibility of an excess of young stars in the solar neighbourhood related to the Gould Belt.

Acknowledgements. This project has been supported by grants from the Deutsche Forschungsgemeinschaft (DFG Schwerpunktprogramm "Physics of star formation"). We would like to thank the staff at Kitt Peak, Calar Alto, and La Silla for their support during observations. This research has made use of the SIMBAD database, operated at CDS, Strasbourg, France

\section{References}

Briceño, C., Hartmann, L. W., Stauffer, J. R., Gagne, M., \& Stern, R. A. 1997, AJ, 113, 740

Cutispoto, G., Pallavicini, R., Kürster, M., \& Rodono, M. A\&A, 297, 764

Cutispoto, G., Pastori, L., Pasquini, L., et al. 2002, A\&A, 384, 491

Dehnen, W., \& Binney, J. J. 1998, MNRAS, 298, 387

de la Reza, R., Torres, C. A. O., Quast, G., Castillo, B. V., \& Vieira, G. L. 1989, ApJ, 343, L61

De Zeeuw, P. T., Hoogerwerf, R., De Bruijne, J. H. J., Brown, A. G. A., \& Blaauw, A. 1999, AJ, 117, 354

Eggen, O. J. 1994, Galactic and Solar System Optical Astrometry, ed. L. V. Morrison, \& G. Gilmore (Cambridge: Cambridge University Press), 191

ESA 1997a, The Tycho Catalogue, ESA SP-1200

ESA 1997b, The Hipparcos Catalogue, ESA SP-1200

Favata, F., Barbera, M., Micela, G., \& Sciortino, S. 1993, A\&A, 277, 428

Fekel, F. C. 1997, PASP, 109, 514
Fisher, N. I., Lewis, T., \& Embleton, B. J. J. 1987, Statistical analysis of spherical data (Cambridge: Cambridge University Press)

Gagne, M., Valenti, J. A., Linsky, J. L., Tagliaferri, G., Covino, S., \& Gudel, M. 1999, ApJ, 515, 423

Gregorio-Hetem, J., Lépine, J. R. D., Quast, G., Torres, C. A. O., \& de la Reza, R. 1992, AJ, 103, 549

Guillout, P., Schmitt, J. H. M. M., Egret, D., et al. 1998, A\&A, 351, 1003

Guillout, P., Sterzik, M. F., Schmitt, J. H. M. M., Motch, C., \& Neuhäuser, R. 1998, A\&A, 337, 113

Haywood, M., Robin, A. C., \& Creze, M. 1997, A\&A, 320, 440

Hearty, T., Magnani, L., Caillault, J. P., et al. 1999, A\&A, 341, 163

Helfand, D. J., Schnee, S., Becker, R. H., White, R. L., \& McMahon, R. G. 1999, AJ, 117, 1568

Henry, G. W., Fekel, F. C., \& Hall, D. S. 1995, AJ, 110, 2926

Hussain, G. A. J., Unruh, Y. C., \& Collier Cameron, A. 1997, MNRAS, 288, 343

Kroupa, P., Tout, C. A., \& Gilmore, G. 1993, MNRAS, 262, 545

Lampton, M., Lieu, R., Schmitt, J. H. M. M., et al. 1997, ApJS, 108, 545

Madsen, S., Dravins, D., \& Lindegren, L. 2002, A\&A, 381, 446

Mamajek, E. E., Lawson, W. A., \& Feigelson, E. D. 1999, ApJ, 516, L77

Mason, K. O., Hassall, B. J. M., \& Bromage, G. E. 1995, MNRAS, 274, 1194

Montes, D., Lopez-Santiago, J., Galvez, M. C., et al. 2001, MNRAS, 328,45

Montes, D., Lopez-Santiago, J., Fernandez-Figueroa, M. J., \& Galvez, M. C. 2001, A\&A, 379, 976

Olsen, E. H. 1994, A\&AS, 104, 429

Osten, R. A., \& Saar, S. H. 1998, MNRAS, 295, 257

Pöppel, W. 1997, FCPh, 18, 1

Pounds, K. A., Allan, D. J., Barber, C., et al. 1993, MNRAS, 260, 77 
Pye, J. P., McGale, P. A., Allan, D. J., et al. 1995, MNRAS, 274, 1165 Rana, N. C. 1991, ARA\&A, 29, 129

Randich, S., Giampapa, M. S., \& Pallavicini, R. 1994, A\&A, 283, 893

Rucinski, S. M., \& Krautter, J. 1983, A\&A, 121, 217

Schmidt-Kaler, Th. 1982, in Landolt-Börnstein, vol. 2b (BerlinHeidelberg: Springer)

Siess, L. E., Dufour, E., \& Forestini, M. 2000, A\&A, 358, 593

Soderblom, D. R., Jones, B. F., Balachandran, S., et al. 1993, AJ, 106, 1059

Sterzik, M. F., \& Schmitt, J. H. M. M. 1997, AJ, 114, 1673

Strassmeier, K. G., Bartus, J., Cutispoto, G., \& Rodono, M. 1997, A\&AS, 125, 11

Tagliaferri, G., Covino, S., Fleming, T. A., et al. 1997, A\&A, 321, 850
Tagliaferri, G., Cutispoto, G., Pallavicini, R., Randich, S., \& Pasquini, L. 1994, A\&A, 285, 271

Torres, C. A. O., Da Silva, L., Quast, G. R., De La Reza, R., \& Jilinski, E. $2000, A J, 120,1410$

Webb, R. A., Zuckerman, B., Platais, I., et al. 1999, ApJ, 512, L63

Wichmann, R., Bastian, U., Krautter, J., Jankovics, I., \& Rucinski, S. M. 1998, MNRAS, 301, L39

Wichmann, R., Covino, E., Alcalá, J. M., et al. 1999, MNRAS, 307, 909

Wichmann, R. 2000, A\&A, 363, 223

Wielen, R. 1971, A\&A, 13, 309

Wielen, R. 1977, A\&A, 60, 263

Zuckerman, B., \& Webb, R. A. 2000, ApJ, 535, 959 